\title{
Numerical Analysis of Four-Wave Mixing between 2 ps mode-locked laser pulses in a Tensile-Strained Bulk SOA
}

\author{
Michael J. Connelly ${ }^{\mathrm{a}}$, Liam P. Barry ${ }^{\mathrm{b}}$, Brendan F. Kennedy ${ }^{\mathrm{c}}$ and Douglas A. Reid ${ }^{\mathrm{b}}$. \\ ${ }^{a}$ Optical Communications Research Group, Dept. Electronic and Computer Engineering, University of \\ Limerick, Limerick, Ireland, E-mail: michael.connelly@ul.ie. \\ ${ }^{\mathrm{b}}$ RINCE, School of Electronic Engineering, Dublin City University, Dublin 9, Ireland. \\ ${ }^{\mathrm{c}}$ Universidad de Santiago, Chile.
}

\begin{abstract}
A numerical model of four-wave mixing between 2-ps pulses in a tensile-strained bulk semiconductor optical amplifier is presented. The model utilizes a modified Schrodinger equation to model the pulse propagation. The Schrodinger equation parameters such as the material gain first and second order dispersion, linewidth enhancement factors and optical loss coefficient are obtained using a previously developed steady-state model. The predicted four-wave mixing pulse characteristics show reasonably good agreement with experimental pulse characteristics obtained using Frequency Resolved Optical Gating.
\end{abstract}

\section{INTRODUCTION}

${ }^{*}$ Semiconductor optical amplifier (SOA) technology has matured to the point where commercial devices are available for use in optical communication systems. SOAs have many functional applications, one of most important of which is wavelength conversion. One of the most promising wavelength conversion mechanisms in an SOA is four-wave mixing (FWM), which is transparent to modulation format and bit rate and has a wide conversion bandwidth. High conversion efficiency can be achieved by using narrow pump and probe pulses. Mathematical models of the FWM process occurring between two optical pulses in an SOA are required in order to design optical subsystems utilizing this process and also to enhance understanding of the FWM process itself.

Most SOA pulse propagation models use a variant of a modified non-linear Schrödinger equation (MSE), which includes non-linearities that are relevant to the pulse time scale. Pulse propagation through an SOA is strongly dependent on the input pulse shape.

The SOA modeled in this paper is a commercially available device manufactured by Kamelian. It has a tensile strained InGaAsP active region sandwiched between two InGaAsP separate-confinement heterostructure layers and operates in the $1.55 \mu \mathrm{m}$ region. The device structure consists of central active region waveguide of width $1.1 \mu \mathrm{m}$, thickness $0.1 \mu \mathrm{m}$ and length $840 \mu \mathrm{m}$. The active region narrows linearly as a lateral taper of length $80 \mu \mathrm{m}$ to width $0.5 \mu \mathrm{m}$ at each end. The tensile strain is used to compensate for the different TE and TM confinement factors in order to achieve polarization independent operation. In this paper we model the copropagation of two $10 \mathrm{GHz}$ repetition rate 2 ps mode-locked

\footnotetext{
* This research was supported by Science Foundation Ireland Investigator Grant 02/IN1/I42.
}

laser pulses. The FWM signal from the SOA model is compared with the pulse temporal profile obtained experimentally using Frequency Resolved Optical Gating (FROG) [1].

\section{PULSE PROPAGATION MODEL}

The MSE used to model the propagation in the SOA of an optical field with complex amplitude $V(z, \tau)$ is given by [2],

$$
\begin{aligned}
& {\left[\frac{\partial}{\partial z}+\frac{\gamma(\tau)}{2}+\left(\frac{\gamma_{2 p}}{2}+i b_{2}\right)|V(z, \tau)|^{2}\right] V(z, \tau)=} \\
& \left\{\frac{1}{2} g_{n}(z, \tau)\left[1 / f(\tau)+i \alpha_{n}(z, \tau)\right]+\frac{1}{2} \Delta g_{T}(z, \tau)\left[1+i \alpha_{T}(z, \tau)\right]\right. \\
& \left.-\left.\frac{i}{2} \frac{\Gamma \partial g_{m}(z, \tau)}{\partial \omega}\right|_{\omega_{0}} \frac{\partial}{\partial \tau}-\left.\frac{1}{4} \frac{\Gamma \partial^{2} g_{m}(z, \tau)}{\partial^{2} \omega}\right|_{\omega_{0}} \frac{\partial^{2}}{\partial \tau^{2}}\right\} V(z, \tau)
\end{aligned}
$$

where the $\tau$ is the local time. In the model we also take into account the spatial and time dependency of the attenuation coefficient $\gamma(\tau)$ and linewidth enhancement factors $\alpha_{N}(\tau)$ (due to carrier density changes) and $\alpha_{T}(\tau)$ (carrier heating). $\gamma_{2}$ is the two-photon absorption (TPA) coefficient . $f(\tau)$ is a function accounting for spectral hole burning. The change in the dynamic gain due to carrier heating is given by

$$
\Delta g_{T}(z, \tau)=-\int_{-\infty}^{\infty} U(s) e^{-s / \tau_{c h}}\left[\begin{array}{l}
h_{1}|V(\tau-s)|^{2} \\
+h_{2}|V(\tau-s)|^{4}
\end{array}\right] d s
$$

$h_{1}$ and $h_{2}$ are phenomenological constants representing the contribution of stimulated emission, free-carrier absorption and TPA to carrier heating. The dynamic gain due to carrier density changes is obtained from the solution to the carrier density rate equation

$$
\frac{d n}{d t}=\frac{\eta I_{e f f}}{e V}-R(n)-\frac{\Gamma}{d W(z)} g_{n}(n)|V(z, \tau)|^{2}
$$




\section{NUSOD 2007}

where $I_{\text {eff }}(z)$ is an effective bias current introduced to account for amplified spontaneous emission. Parameters in the MSE such as the gain coefficient dispersion, linewidth enhancement factors and material gain coefficient were obtained using the steady-state model and parameter extraction algorithm [3]. Reasonable estimates of the MSE phenomenological constants were obtained by investigating the propagation of a pulse stream through the SOA using FROG and then using the MSE and Levenberg-Marquardt parameter extraction technique [4].

\section{NUMERICAL ALGORITHM}

The SOA model equations cannot be solved analytically, so a numerical solution is required. The MSE can be solved by using the finite-difference beam propagation method described in [1]. The modified-Euler finite difference technique is used to solve the carrier density rate equation. To determine the solution in the FWM case, the input pulse amplitude can be expressed as

$$
V_{\text {in }}(t)=V_{\text {pump }}(t)+V_{\text {probe }}(t) \exp (-j \Delta \omega t)
$$

where $V_{\text {pump }}(t)$ and $V_{\text {probe }}(t)$ are the complex pump and probe pulse amplitudes. $\Delta \omega$ is the detuning between the pump and probe.

\section{SIMULATIONS AND EXPERIMENT}

In the experiment, two $10 \mathrm{GHz}$ repetition rate optical pulse streams were obtained from two actively mode-locked wavelength tunable lasers, combined and amplified by the SOA. The input pump and probe pulse peak powers were 165 $\mathrm{mW}$ and $10 \mathrm{~mW}$ respectively. The resulting FWM signal was filtered and then analyzed using FROG.

The input to the model was the actual measured complex pulse amplitude. A feature of the pulses is that pedestals are present on both the leading and trailing edges of the main pulse. The FWM pulse shape was investigated as the detuning between the pump and probe was varied. The FROG experimental results, shown in Fig. 1, show that as the detuning increases the relative importance of the pedestals increases. These pedestals can lead to significant intersymbol interference when the wavelength converted pulse is used in high-speed optical time division multiplexed systems.

The simulated FWM signal, for three detuning levels is shown in Fig. 2. There is reasonably good agreement between experiment and simulations. The simulations successfully predict that the pedestals on the FWM signal are greatly enhanced compared to the pedestals on the input probe signal.

As can be seen from Fig. 1 the FWM signal has a complex shape, which indicates the complexity of the underlying physical processes in the SOA. The principle uncertainties in comparing the experimental results to the model predictions is the poor accuracy of the FROG technique at power levels $>30$
$\mathrm{dB}$ below the peak power and the precise effect of the optical filter used to select the FWM signal especially at low detuning.

Further results including a more detailed description of the numerical algorithms and more extensive simulations will be presented at the conference.

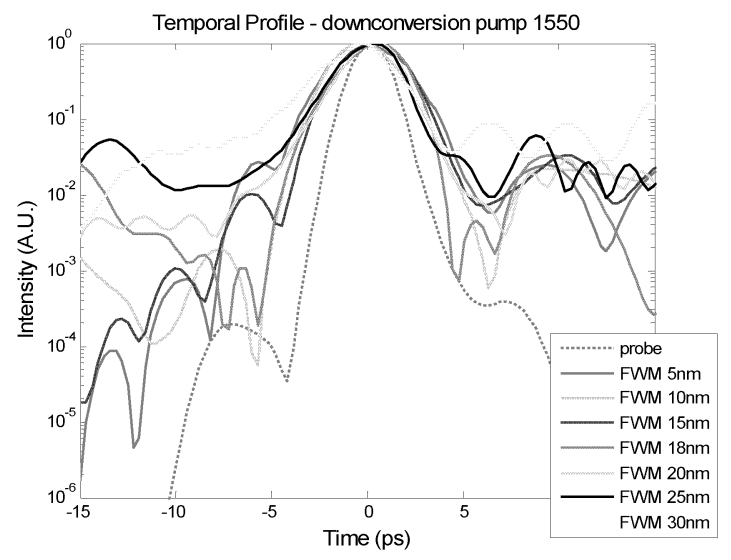

Fig. 1. FROG recovered FWM pulse temporal profiles for various down conversion detuning showing pedestal level increasing with increasing detuning. The pump pulse shape is similar to the probe.

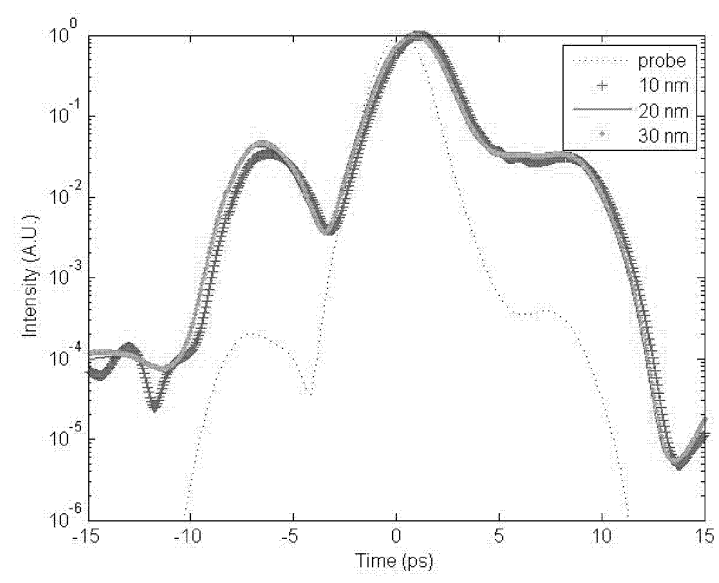

Fig. 2. Simulated FWM pulse temporal profiles for various down conversion detuning.

\section{REFERENCES}

[1] A.M Clarke, M.J. Connelly, P.M. Anandarajah, L.P. Barry and D.A. Reid, "Investigation of pulse pedestal and dynamic chirp formation on picosecond pulses after propagation through a semiconductor optical amplifier," IEEE Photon Tech. Lett., , vol. 17, pp. 1800-1802, 2005.

[2] N.K. Das, Y. Yamayoshi and H. Kawaguchi,"Analysis of basic fourwave mixing characteristics in a semiconductor optical amplifier by the finite-difference beam propagation method," IEEE J. Quantum Electron., vol. 36, pp. 1184, 2000.

[3] M.J. Connelly, "Wide-band Steady-State Numerical Model and Parameter Extraction of a Tensile-Strained Bulk Semiconductor Optical amplifier," IEEE J. Quantum Electron. vol. 43, pp. 47-56, 2007.

[4] M.J. Connelly, A.M. Clark, P.M. Anandarajah and L.P. Barry, "Numerical analysis of picosecond pulse propagation in a tensile-strained semiconductor optical amplifier with parameter extraction using frequency resolved optical gating", paper no. ThG09, $13^{\text {th }}$ European Conference on Integrated Optics, Copenhagen, 2007. 